\title{
CLIMA ORGANIZACIONAL Y SU INFLUENCIA EN LA ESTRUCTURACIÓN DEL PLAN ESTRATÉGICO EN EL INSTITUTO SUPERIOR PEDAGÓGICO PRIVADO PAULO FREIRE DEL DISTRITO DE COMAS
}

"ORGANIZATIONAL CLIMATE AND ITS INFLUENCE ON THE STRUCTURE OF THE STRATEGIC PLAN FOR HIGHER EDUCATIONAL INSTITUTE PRIVATE PAULO FREIRE LOCATED IN THE DISTRICT OF COMAS"

\author{
María Celina Huamán Mejía* \\ praxisquality@gmail.com \\ Santiago Inga Portal*** \\ santiago.inga@gmail.com
}

\begin{abstract}
RESUMEN
En este artículo, se presentan los resultados de la investigación sobre la influencia del clima organizacional en la estructuración del Plan Estratégico Institucional del Instituto Superior Pedagógico Privado Paulo Freire.

El análisis de esta organización permite establecer la relación del clima organizacional, con respecto a la satisfacción de los actores de la institución, según las dimensiones de Litwin y Stringer, y el plan estratégico, con miras a mejorar sus procesos para lograr ser una institución acreditada y reconocida en el país.
\end{abstract}

Palabras claves: Clima organizacional, plan estratégico, dimensiones.

\begin{abstract}
The results of research on the influence of organizational climate in the structuring of the Strategic Plan in the Higher Pedagogical Institute Paulo Freire Private presented in this article.

The analysis of this organization allows establishing the relationship of organizational climate, with respect to the satisfaction of the stakeholders of the institution according to the dimensions of Litwin and Stringer; and the Strategic Plan, to improve their processes in order to become an accredited and recognized institution in the country.
\end{abstract}

Keywords: Organizational Climate, Strategic Plan, Dimensions.

\footnotetext{
* Magíster en Administración. Lic. Administración. Evaluadora del Premio Nacional a la Calidad-RGPM. Auditor Líder IRCA ISO 9001. (TÜV Rheinland). Diplomada Internacional en Lean Six Sigma Black Belt (U. Pacífico-Lean Six Sigma Institute). Diplomada en Implementación y Auditoría de Sistemas Integrados de Gestión de la Calidad, Ambiental y Seguridad y Salud Ocupacional. (Instituto para la Calidad PUCP). Especialista en Gestión de la Calidad en Educación (Instituto para la Calidad PUCP). Consultora y asesora en Calidad en pre y posgrado. ** Licenciado en Psicología. Bachiller en Administración.
} 


\section{INTRODUCCIÓN}

En los últimos años, las instituciones educativas han ido aplicando diversas herramientas de gestión que permiten mejorar la calidad en todos los procesos que desarrollan en sus instituciones. Esta tendencia es a nivel mundial y existen diversos organismos que se encargan de acreditar a las instituciones educativas.

En nuestro país, el Sistema Nacional de Evaluación Acreditación y Certificación de la Calidad Educativa (SINEACE) es el conjunto de organismos, normas y procedimientos destinados a establecer los criterios, estándares y procesos de evaluación, acreditación y certificación a fin de asegurar los niveles básicos de calidad que deben brindar las instituciones educativas. Este sistema cuenta con órganos operadores encargados de garantizar la calidad educativa en el ámbito de su competencia: el primero es el Instituto Peruano de Evaluación, Acreditación y Certificación de la Calidad de la Educación Básica (IPEBA), con competencia en las instituciones educativas de educación básica y técnico-productiva; el segundo es el Consejo de Evaluación, Acreditación y Certificación de la Calidad de la Educación Superior No Universitaria (CONEACES), con competencia en las instituciones de educación superior no universitaria; y el último es el Consejo de Evaluación, Acreditación y Certificación de la Calidad de la Educación Superior Universitaria (CONEAU), con competencia en las instituciones de educación superior universitaria.

Con el fin de alinearse a una adecuada gestión, la organización debe comenzar por realizar el Plan Estratégico Institucional, el cual según la normativa del CONEACES, se denomina Proyecto Educativo Institucional. Este proyecto es el primer factor de la dimensión gestión institucional. El segundo factor se denomina organización y gestión administrativa, el cual contiene, en uno de sus indicadores, la promoción de un clima organizacional que conlleve la excelencia académica, por lo cual la opinión de los grupos de interés es importante para establecer una cultura de calidad.

Este artículo busca encontrar la influencia del clima organizacional en la estructuración del Plan Estratégico Institucional, dado que son los pilares en el aseguramiento de la calidad educativa del Instituto Superior Pedagógico Privado Paulo Freire.

\section{PLANTEAMIENTO DEL PROBLEMA}

Con el fin de alinearse a los estándares de calidad exigidos en la actualidad, el Instituto Superior Pedagógico Privado Paulo Freire ha encontrado dificultades en realizar un Plan Estratégico Institucional, por lo que surge la necesidad de analizar la satisfacción de sus principales actores: docentes, personal administrativo y alumnos, expresado en el clima organizacional. Por ello, el problema que buscamos solucionar es:

- ¿Cómo afecta el clima organizacional en la estructuración del Plan Estratégico del Instituto Superior Pedagógico Privado Paulo Freire?

\section{OBJETIVOS}

El objetivo de la investigación consiste en:

- Establecer y analizar la influencia del clima organizacional en la estructuración del Plan Estratégico del Instituto Superior Pedagógico Privado Paulo Freire.

\section{JUSTIFICACIÓN}

El clima organizacional es un aspecto importante en la relación entre personas y organizaciones; además, está íntimamente relacionado con la motivación de los miembros de una organización. Por ello, al haber una motivación elevada, el clima organizacional tiende a ser elevado, manifestándose relaciones de satisfacción, ánimo, interés y colaboración. De esta manera, el contar con un adecuado clima organizacional permite tener resultados exitosos en una organización, logrando la participación de todas las personas involucradas con el fin de ayudar a la gestión de las instituciones y enrumbarlas a obtener calidad en sus procesos con el fin de certificarse o acreditarse, generando un reconocimiento y permitiéndoles dar un producto o servicio óptimo a sus clientes o usuarios.

En el caso del Instituto Superior Pedagógico Privado Paulo Freire, el análisis del clima organizacional permitirá establecer los lineamientos bases para la elaboración del plan estratégico y tomar medidas correctivas si es necesario.

\section{HIPÓTESIS}

La hipótesis planteada es la siguiente:

- El clima organizacional elevado afecta de forma positiva a la estructuración del Plan Estratégico del Instituto Superior Pedagógico Privado Paulo Freire. 


\section{METAS ESPECÍFICAS}

Las metas consisten en:

- Lograr establecer la relación entre el clima organizacional y la estructuración del Plan Estratégico del Instituto Superior Pedagógico Privado Paulo Freire.

- Medir el grado de motivación de los principales actores del Instituto Superior Pedagógico Privado Paulo Freire.

\section{MARCO TEÓRICO DEL TRABAJO}

\section{Clima organizacional \\ Definición}

El clima organizacional es un término que se planteó en el década de los sesenta junto con la aparición del desarrollo organizacional y la aplicación de la teoría de sistemas al estudio de las organizaciones. (Rodríguez, 1999) Así, las definiciones que los investigadores han propuesto para determinar que entienden por clima organizacional han evolucionado a través de distintas aproximaciones conceptuales (Chiang Vega, Martín Rodrigo, \& Núñez Partido, 2010).

Los primeros investigadores resaltaron las propiedades o características organizacionales (Forehand y Gilmer, 1964). Luego se dio mayor relevancia a las representaciones cognitivas e interpretaciones, en la que los factores individuales son los determinantes (James y Jones, 1974). Más tarde, se consideró el clima como un conjunto de percepciones fundamentales o globales en que se considera la interacción entre la persona y situación (Schneider y Reichers, 1990).

Los últimos estudiosos del tema nos muestran una definición más amplia del término. Entre algunas de ellas tenemos:

- El clima organizacional determina la forma en que un individuo percibe su trabajo, su rendimiento, su productividad, su satisfacción, etc. (Martínez, 2003).

- El clima organizacional, se refiere al ambiente propio de la organización, producido y percibido por el individuo de acuerdo a las condiciones que encuentra en su proceso de interacción social y en la estructura organizacional que se expresa por variables (Méndez Álvarez, 2006).
- El clima organizacional expresa la influencia del ambiente sobre la motivación de los participantes, es decir, se puede describir como la cualidad o propiedad del ambiente organizacional que perciben o experimentan sus miembros y que influye en su conducta (Chiavenato, 2007)

Como conclusión podemos decir que el clima organizacional es la identificación de características que hacen los individuos que componen una organización y que influyen en su comportamiento. (García Solarte, 2009).

\section{Herramientas o métodos de diagnóstico del clima organizacional}

Existen diversas herramientas y métodos propuestos por diferentes estudiosos del clima organizacional (García Solarte, 2009). Entre los principales se encuentran:

- El cuestionario de Litwin y Stringer: Este instrumento se desarrolla con el fin de identificar las percepciones (subjetivas) de los individuos y su comportamiento en el ámbito de la organización. Las dimensiones que utilizaron son: estructura organizacional del trabajo, responsabilidad, recompensa, riesgo, apoyo y calidez, estándares, conflicto e identidad lealtad. El cuestionario tiene 50 ítems, con escala de rangos que va desde completamente de acuerdo hasta completamente en desacuerdo.

- El cuestionario de Rensis Likert: La herramienta utilizada por Likert fue diseñada para medir la naturaleza del sistema de gestión y los clasifica en cuatro grupos: sistema I autoritarismo explotador, el sistema II autoritarismo paternalista, el sistema III consultivo y el sistema IV participación en grupo. Los sistemas I y II corresponden a un clima cerrado, donde existe una estructura rígida, por lo que el clima es desfavorable; por otro lado, los sistemas III y IV corresponden a un clima abierto con una estructura flexible, lo que propicia un clima favorable dentro de la organización.

- Modelo de medición de John Sudarsky Test de Clima Organizacional (TECLA): Este modelo plantea en su estudio las siguientes dimensiones del clima organizacional: Conformidad, responsabilidad, normas de excelencia, recompensa, claridad organizacional, calor y apoyo, seguridad, y salario. 
El instrumento de medición del clima organizacional utilizado es un cuestionario de 90 preguntas de falso o verdadero, incluidas varias preguntas de control.

- Cuestionario de Halpin y Crofts: Se basa en ocho dimensiones, las cuales fueron determinadas a través de un estudio realizado en una escuela pública. de esas ocho dimensiones, cuatro apoyaban al cuerpo docente y cuatro al comportamiento del director. Estas dimensiones son: desempeño, implicación del personal docente en su trabajo, obstáculos; sentimiento del personal docente al realizar las tareas rutinarias, intimidad: percepción del personal docente relacionado con la posibilidad de sostener relaciones amistosas con sus iguales; espíritu: satisfacción de las necesidades sociales de los docentes; actitud distante: comportamientos formales e informales del director, donde prefiere atenerse a las normas establecidas antes de entrar a una relación con sus docentes; importancia de la producción; comportamientos autoritarios y centrados en la tarea del director; confianza, esfuerzos del director para motivar al personal docente y consideración: referido al comportamiento del director que intenta tratar al personal docente de la manera más humana (Carbajal Peña, 2000).

\section{Plan estratégico}

\section{Definición}

El plan estratégico tiene diferentes explicaciones debido a la variedad de elementos que lo involucran. Algunas ideas importantes son:

- El plan estratégico consiste en el conjunto de análisis, decisiones y acciones que una organización lleva a cabo para crear y mantener ventajas comparativas sostenibles (Dess, Lumpkin, \& Taylor, 2003).

- El plan es, en esencia, una secuencia de decisiones fundamentales, las que eventualmente deberán convertirse también en una secuencia de acciones (Díaz, 2005).

- El plan estratégico constituye la herramienta en la que la alta dirección recoge las decisiones estratégicas corporativas que ha adoptado hoy, en referencia a lo que hará en los tres próximos años (Sainz de Vicuña Ancín, 2012).
Como conclusión, se entiende que el plan estratégico es un documento que contiene las estrategias que desarrollará una organización en un periodo de tiempo determinado.

\section{Desarrollo del plan estratégico}

El plan estratégico, cuenta con tres pilares fundamentales: diseñar objetivos de negocio, diseñar estrategias de negocio y diseñar actividades de mejora (Álvarez Torres, 2006).

- Objetivos de negocio: Son el conjunto de resultados cuantitativos más importantes para la organización. Cada objetivo tiene una meta anual que obliga a la organización a trabajar unidos para su cumplimiento. La redacción de los objetivos de negocio es un verbo de acción en infinitivo, una meta medible, cuantificable, retadora, realista y alcanzable en un periodo de tiempo.

- Estrategias de negocio: Son el conjunto de conceptos generales que le ayudan a la organización a alcanzar sus objetivos de negocio. La estrategia debe ser un concepto que englobe a un conjunto de actividades. La forma más efectiva de diseñar estrategias es recopilar y analizar la información relativa a cada uno de los grupos de interés de una organización.

- Actividades de mejora: Son el conjunto de acciones específicas a desarrollar durante el año. Cada actividad de mejora puede ser realizada una o varias veces al año. Todas las actividades de mejora deben tener definido un responsable y fecha de ejecución, siendo alineadas a las estrategias planteadas anteriormente.

\section{MÉTODO}

El estudio es de carácter descriptivo, ya que se identifica y describe las características esenciales de los factores de estudio. Además es de tipo aplicado, ya que se limita a conocer cómo influye el clima organizacional en la elaboración del plan estratégico.

Las técnicas de recolección de datos fueron cuestionarios y entrevistas, siendo el diseño de las escalas de medición elaboradas siguiendo el modelo del cuestionario de Litwin y Stringer, el cual se basa en nueve dimensiones: estructura, responsabilidad, recompensa, riesgo, calidez, apoyo, normas, conflicto e identidad. 
El universo de la investigación consiste en los principales actores del Instituto Superior Pedagógico Privado Paulo Freire, es decir, la suma de docentes, alumnos y personal administrativo, siendo un total de 302 personas. Hallando el número de muestra a encuestar, se obtuvo 64 personas, con un margen de error de $5 \%$, nivel de confianza $95 \%$ y $\mathrm{p}=90$ (Cuadro $\left.\mathrm{N}^{\circ} 01\right)$.

\section{Cuadro $\mathrm{N}^{\circ} 01$}

Distribución de la muestra

\begin{tabular}{|c|c|c|c|}
\hline Actores & $\begin{array}{c}\text { Número } \\
\text { Total }\end{array}$ & $\begin{array}{c}\text { Participación } \% \\
\text { de los actores }\end{array}$ & $\begin{array}{c}\text { Muestra según } \\
\text { participación } \%\end{array}$ \\
\hline Alumnos & 205 & $68 \%$ & 44 \\
\hline Docentes & 67 & $22 \%$ & 14 \\
\hline $\begin{array}{c}\text { Personal ad- } \\
\text { ministrativo }\end{array}$ & 30 & $10 \%$ & 6 \\
\hline Total & 302 & $100 \%$ & 64 \\
\hline
\end{tabular}

Fuente: Elaboración propia.

\section{RESULTADOS}

Los resultados se presentan según las nueve dimensiones del clima organizacional.

\section{Estructura}

El gráfico $\mathrm{N}^{\circ} 01$ muestra en líneas generales la aprobación de los actores principales de la institución, en cuanto a la percepción de un adecuado manejo de las reglas, procedimientos y trámites que realiza el instituto. En el caso del personal administrativo, se expresa en un $83 \%$, mientras que los docentes cuentan con un $43 \%$ de acuerdo y los alumnos con un $34 \%$ muy de acuerdo.

\section{Gráfico $\mathrm{N}^{\circ} 01$}

Percepción del adecuado manejo de las reglas, procedimientos y trámites

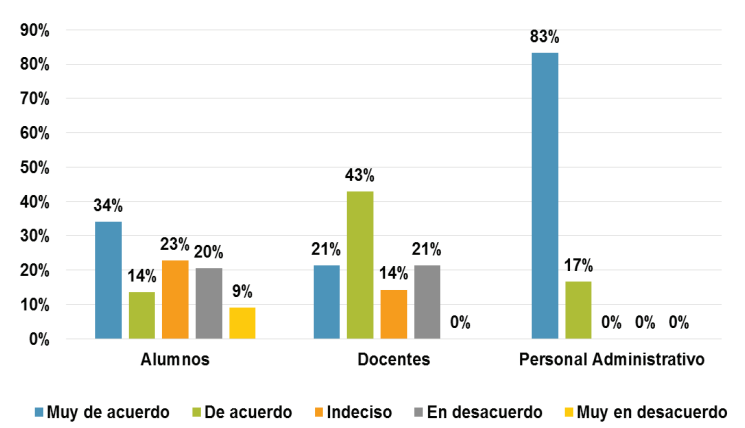

Fuente: Elaboración propia.

\section{Responsabilidad}

En el gráfico $\mathrm{N}^{\circ} 02$ se indica que en promedio la mitad de los actores consideran que tienen autonomía al tomar una decisión, ya sea en sus labores como docentes o personal administrativo o como alumnos. Esta afirmación se expresa en el $43 \%$ muy de acuerdo de los alumnos, el 36\% de acuerdo de los docentes y el $50 \%$ muy de acuerdo del personal administrativo.

\section{Gráfico $\mathrm{N}^{\circ} 02$}

Percepción de la autonomía en la toma de decisiones

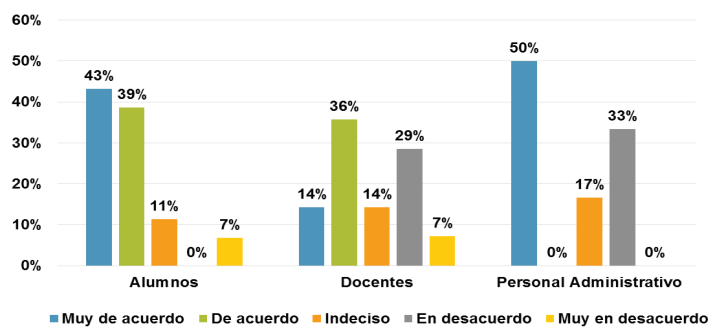

Fuente: Elaboración Propia

\section{Recompensa}

En cuanto a la recompensa, los alumnos se encuentran desmotivados, lo que se expresa en el $50 \%$ que se encuentra en desacuerdo, mientras que los docentes muestran un $43 \%$ que se encuentra de acuerdo y el personal administrativo también expresa su conformidad con un $50 \%$ (Gráfico $\left.\mathrm{N}^{\circ} 03\right)$.

\section{Gráfico $\mathrm{N}^{\circ} 03$}

Percepción de la recompensa o motivación

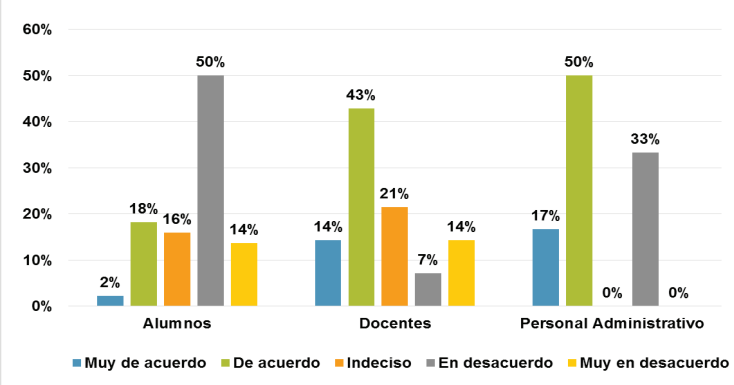

Fuente: Elaboración propia.

\section{Riesgo}

En cuanto al riesgo, entendido por la capacidad de enfrentar nuevos desafíos por parte de la organización, se observa una percepción 
diferente por parte de los alumnos, que en un $34 \%$ expresan estar en desacuerdo, mientras que un $43 \%$ de docentes y un 50\% de personal administrativo consideran estar de acuerdo (Gráfico N04).

\section{Gráfico $\mathrm{N}^{\circ} 04$}

Percepción de la capacidad de enfrentar nuevos desafíos

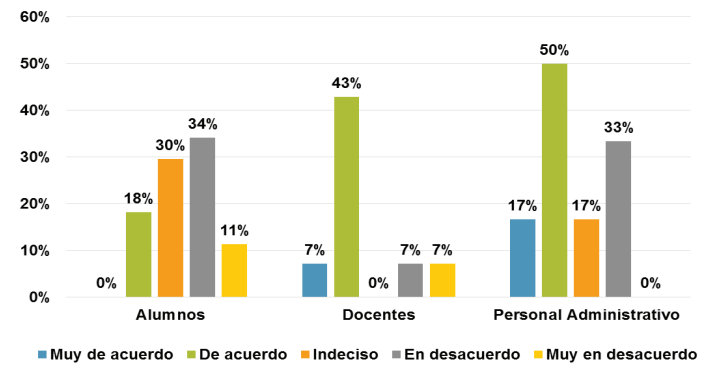

Fuente: Elaboración propia.

\section{Calidez}

Respecto a la calidez, se consulta sobre la percepción de la colaboración en la organización, a lo que los alumnos indican estar de acuerdo en un $30 \%$, al igual que los docentes con un 50\% y los administrativos en un 67\% (gráfico $\mathrm{N}^{\circ} 05$ ).

\section{Gráfico $\mathrm{N}^{\circ} 05$}

\section{Percepción de la colaboración en la organización}

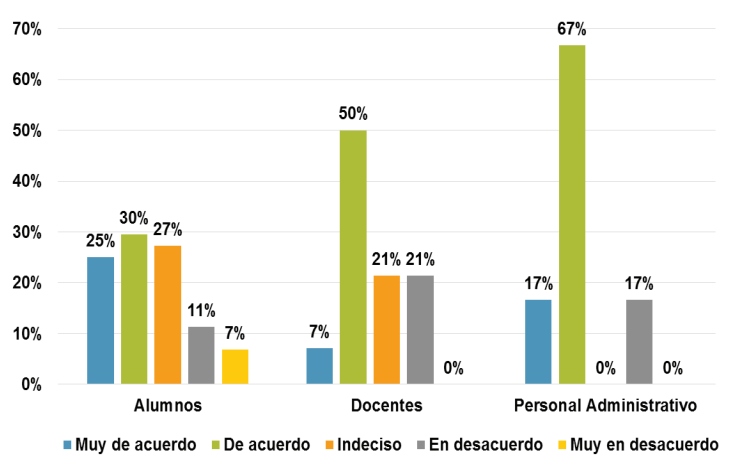

Fuente: Elaboración propia.

\section{Apoyo}

El apoyo es la percepción de ayuda en la organización. Los alumnos indican estar en desacuerdo en un 34\%, al igual que los docentes con un 36\%, mientras que el personal administrativo muestra indicadores positivos en un $100 \%$ (gráfico $\mathrm{N}^{\circ} 06$ ).

\section{Gráfico $\mathrm{N}^{\circ} 06$}

\section{Percepción de apoyo en la organización}

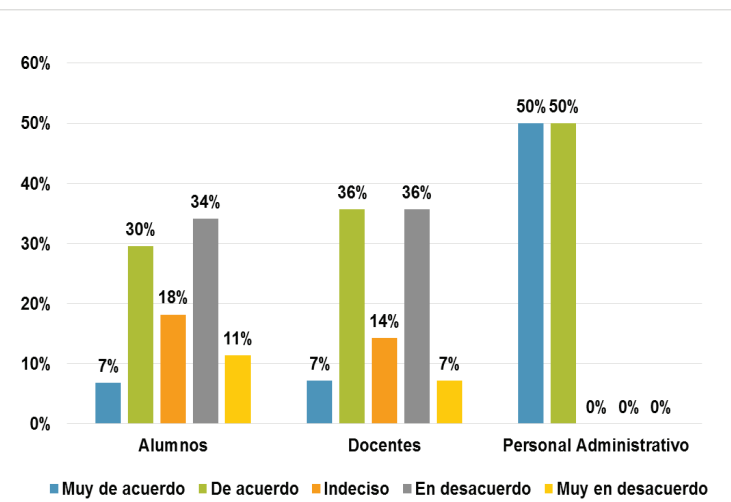

Fuente: Elaboración propia.

\section{Normas}

En cuanto a las normas, lo que los alumnos indican estar de acuerdo en un 36\% es con respecto al énfasis que da la organización a las normas de rendimiento, al igual que los docentes en un $71 \%$, mientras que el personal administrativo muestra estar de acuerdo y en desacuerdo en un $33 \%$ en cada caso (gráfico $\mathrm{N}^{\circ} 07$ ).

\section{Gráfico $\mathrm{N}^{\circ} 07$}

Percepción de apoyo en la organización

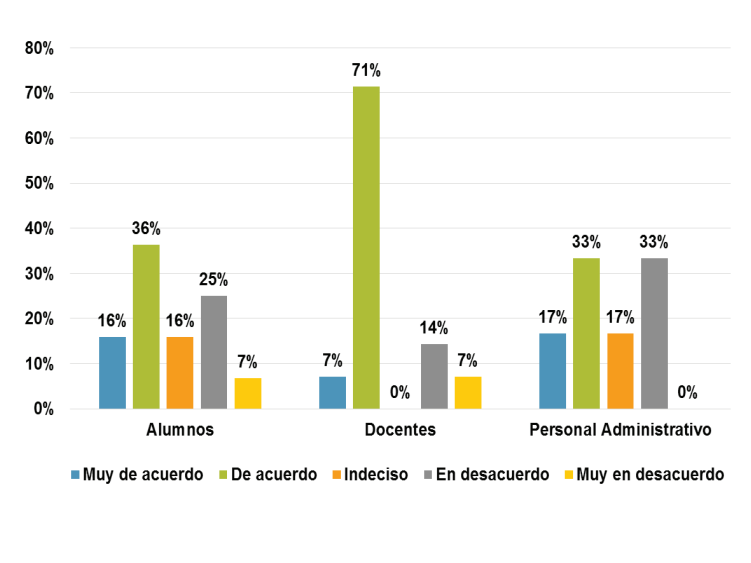

Fuente: Elaboración propia.

\section{Conflicto}

El conflicto se refiere a la capacidad de aceptar las opiniones discrepantes, enfrentar problemas y dar soluciones. En este caso, los alumnos indican estar en desacuerdo en un $41 \%$, mientras que los docentes expresan su indecisión con un 57\%. El personal administrativo muestra estar muy de acuerdo y de acuerdo en un 33\% en cada caso (gráfico $\mathrm{N}^{\circ} 08$ ). 
Gráfico №8 08

Percepción de apoyo en la organización

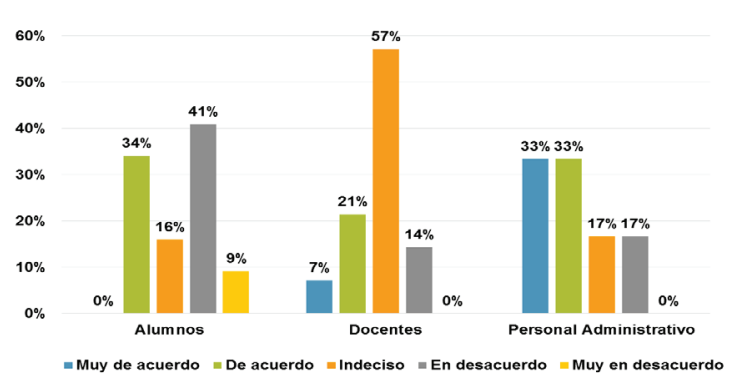

Fuente: Elaboración propia.

\section{Identidad}

En el caso de la identidad, se refiere a la percepción de pertenecer y compartir la identidad personal con la organización, además de conocer los objetivos del instituto. Los alumnos indican estar en $34 \%$ indecisos, mientras que los docentes expresan estar muy de acuerdo y de acuerdo con un $36 \%$ en cada caso, y el personal administrativo muestra estar indeciso y en desacuerdo en un 33\% respectivamente (gráfico $\mathrm{N}^{\circ} 09$ )

\section{Gráfico Nº9}

\section{Percepción de apoyo en la organización}

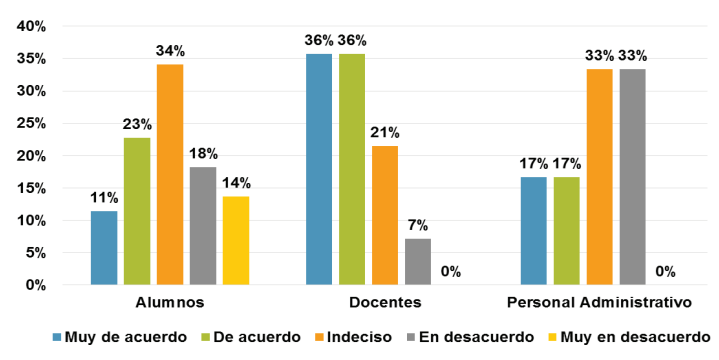

Fuente: Elaboración propia.

\section{DISCUSIÓN}

En el Instituto Superior Pedagógico Privado Paulo Freire, se observa que existe una mayor insatisfacción de los alumnos expresada en la dimensión de recompensa, debido a la falta de motivación que perciben, y en la dimensión de conflicto, en la que consideran que es difícil la aceptación de opiniones discrepantes.

También, al analizar las cifras de los docentes, se observa que la mayor cifra de inconformidad se encuentra en la poca percepción de aceptación de opiniones discrepantes, enfrentar problemas y darles solución, mayormente en el caso de las relaciones con sus encargados en la parte administrativa.

Por último, en el caso del personal administrativo, la principal insatisfacción se expresa en la falta de identidad personal con la organización, así como conocer los objetivos que persigue la organización.

Todos los factores de análisis presentados indican la poca conformidad con los lineamientos de gestión de la institución; además, al sentir que no se aceptan las opiniones, tanto de alumnos como de docentes, sumada la falta de identidad del personal administrativo, conlleva obtener un bajo clima organizacional, lo cual influye en la adecuada estructuración del plan estratégico, debido a que no se hacen partícipes a todos los actores de la institución.

\section{CONCLUSIONES}

Las conclusiones del estudio son:

- El clima organizacional tiene una gran influencia en la estructuración del Plan Estratégico del Instituto Superior Pedagógico Privado Paulo Freire, dado que al no tomar en cuenta ciertos factores de importancia para las personas, en este caso algunas dimensiones, no se logra plantear adecuadamente los objetivos estratégicos $y$, por ende, no existe un apoyo y un acompañamiento al logro de ellos.

- El grado de motivación de los principales actores del Instituto Superior Pedagógico Privado Paulo Freire, en relación con el clima organizacional, es relativamente positivo en ciertas dimensiones, pero en aquellas que tienen una estrecha relación con una adecuada gestión existen brechas que mejorar.

\section{AGRADECIMIENTOS}

Agradezco a la Facultad de Ciencias Administrativas, especialmente al director del Instituto de Investigación, Dr. Juan Barreda, y a las principales autoridades de la facultad, por brindar la oportunidad de publicar este artículo y aportar al conocimiento científico aplicativo de la carrera profesional de Administración. 


\section{REFERENCIAS BIBLIOGRÁFICAS}

ÁLVAREZ TORRES, M. (2006): "Manual de Planeacion Estratégica". México DF: Panorama Editorial.

CARBAJAL PEÑA, G. (2000): "Importancia de la Cultura y Clima Organizacional como factores determinantes en la eficacia del personal civil en el contexto militar". Lima: Universidad Santa María.

CHIANG VEGA, M., Martín Rodrigo, M. J., \& Núñez Partido, A. (2010): "Relaciones entre el clima organizacional y la satisfacción laboral”. Madrid: Universidad Pontifica Comillas.

CHIAVENATO, I. (2007): "Administración de Recursos Humanos". México DF: McGraw-Hill.

DESS, G., Lumpkin, G. T., \& Taylor, M. (2003): "Dirección Estratégica”. España: McGraw-Hill.
DÍAZ, L. F. (2005): "Análisis y planeamiento". San José: EUNED.

GARCÍA SOLARTE, M. (2009): "Clima Organizacional y su diagnóstico: una aproximación conceptual". Cuadernos de Administración, 43-62.

MARTÍNEZ, M. (2003): "La gestión empresarial: equilibrando objetivos y valores”. México DF: Ediciones Díaz de Santos.

MÉNDEZ ÁLVAREZ, C. E. (2006): "Clima Organizacional en Colombia”. Centro Editorial de la Universidad del Rosario.

RODRÍGUEZ, D. (1999): "Diagnóstico Organizacional”. México. D.F.: Editorial Alfaomega.

SAINZ DE VICUÑA ANCÍN, J. M. (2012): "El plan estratégico en la práctica”. Madrid: ESIC Editorial. 